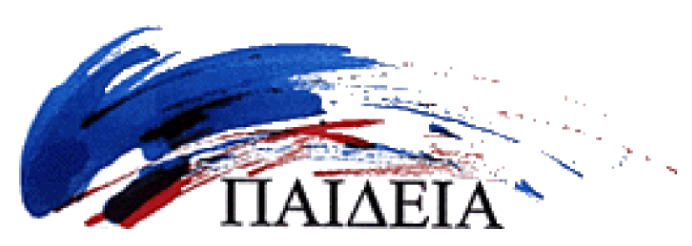

Philosophy of Mathematics

\title{
Translation, Quotation and Truth
}

\author{
Roger Wertheimer \\ wertheim@csulb.edu
}

\begin{abstract}
If logical truth is truth due solely to syntactic form, then mathematics is distinct from logic, even if all mathematical truths are derivable from definitions and logical principles. This is often obscured by the plausibility of the Synonymy Substitution Principle that is implicit in the Fregean conception of analyticity: viz., that synonyms are intersubstitutable without altering sentence sense. Now, unlike logical truth, mathematical truth is not due to syntax, so synonym interchange in mathematical truths preserves sentence syntax, sense, and mathematical necessity. Mathematical necessity, therefore, differs from both logical and lexical necessity.
\end{abstract}

Alonzo Church's Translation Test is supposed to evaluate alleged necessary equivalences. The basic idea is that (correct) translation is meaning preserving, so if some disparity between the alleged equivalents appears under translation, the sentences are inequivalent. The Test translations are essentially synonym substitutions. The translation sentence is to have the syntax of the original, and to differ only morphemically, by synonym subbing. Supposedly, whether the translations are inter-language or intra-language has no logical import. Either way, the underlying and seemingly unassailable principle is that synonym subbing transmits sentence sense. This Synonym Substitution Principle extends to subbing of coreferring proper names by taking their meaning to be their reference.

The Test's derivation from this Principle should spark suspicions for two reasons. First, the Principle seems to entail the Test's inutility, for, presumably, any disparity appearing under translation but not appearing in the original must be a discrepancy in and of the translation. If the Principle is platitudinous, the Test should be profitless. This isn't the old Paradox of Analysis. Synonymies aren't analyses. Genuine analyses are translanguistic necessary 
truths knowable a priori; synonymies are contingent notational equivalences knowable only a posteriori. Unlike analysis, the assumption that translation can be informative for a competent bilingual needs justification.

The Test's pet targets are peculiarly Carnapian theses: e.g., that belief attributions like Mother believes Roger is nearby are semantic equivalents of speech attributions like Mother accepts "Roger is nearby". The Test's plausibility and popularity may owe much to its confirming incredulity about independently refutable theses. We knew preChurch that my dog Mother's believing her master is nearby doesn't entail any wagging at an English sentence.

Another curiosity in the Test is that translation seems to reveal disparities not otherwise evident solely with talk about talk and thought, in the relation between direct and indirect discourse. The Test gets touted only with instantiations of alleged logical or analytical principles wherein, allegedly, a quotation embedding sentence mutually entails a quotationless sentence. When a quotation names its enquoted expression by enquoting it, any intraquotation substitution ipso facto changes both the name and its referent. So no quotational name of an expression is translatable as the expression named may be. So if the Test works, it's only when and because the Principle entailing it doesn't. The Test is trading on the Principle's only exception that Churchfolk admit, intraquotation substitution.

The Test turns on translating Bqsi by Bqsg

$\mathrm{B}$ : Blood is red

Bg: Blut ist rot

Bqsi: "Blood is red" says that blood is red

Bqsg: "Blood is red" sagt dass Blut is rot

The Test severs the internal relation between a quotational name and its enquoted referent. It presumes such severance lacks semantic salience. The Test denies that QSI sentences are logical truths, syntactically unlike the extralogical contingencies of other QS sentences.

QS: "..." means (says, designates, etc.)

QSI: "..." means ...

And the Test assumes that the Synonym Substitution Principle applies to logical sentences just as it does to nonlogical sentences. 
That supposition may seem innocent, but only blind fealty to a semantic theory could silence the sound of nonsynonymy netted by nonuniform subbing of synonymous terms in logical truths:

\section{LL: A lawyer is a lawyer.}

\section{AL: An attorney is a lawyer.}

AgL: An Anwalt is a lawyer

MM: If she was married, she was married

MW: If she was married, she was wed

Just as plainly, logical sentences are not used interchangeably with their synomic correlates. Still, the Principle can seem so self-evident that theorists may assume the data of intuition and usage must be illusory . until someone explains why and how there could be nonsynonymy here when the factual content (the objects denoted, the properties predicated) is identical.

That mystery dissipates by appreciating that (1) sentence meaning depends on syntax no less than on lexical meanings, and (2) intercepting . i.e.: nonuniformly replacing a recurrence of a term pivotal in the truth securing syntax of a logical sentence . is a categorical syntactic switch. Intercepting converts a logical sentence into an extralogical one, thereby transforming its inferential relations with all other sentences. Term recurrence is essential to logical truth, because it's the sole strictly syntactic device enforcing necessary coextension of terms, thereby securing necessary truth in a sentence appropriately structured by logical constants. The semantic structure of logical syntax must be special, because term meanings become irrelevant to truth.

Synonymous terms are also ipso facto coextensive, so synonym intercepting transmits truth in all possible worlds. But this "necessity" is "lexical", not logical, secured not by syntax, but by notational contingency. Expectably, synomic interceptions are most naturally said and read as expressions of the notational contingency. When used to inform an audience of some fact, AL ("An attorney is a lawyer") expresses metalinguistic propositions it presupposes:

aqsl: "Attorney" means lawyer

aqqsl: "Attorney" means what "lawyer" means 
Certainly, the sentence can be meant and understood objectually, as a claim about (an) attorney(s), and it is only as such that its truth is necessary. Yet this objectual necessity shares the same (lack of) extralinguistic content and entailments as the metalinguistic contingencies explaining it.

Denial of interceptional nonsynonymy is driven by preconceptions of undeniable power that have shaped Analytical thought since Locke trashed as "trifling" the essentialist truths the Ancients were after. A net of misconceptions needs unraveling. One might confuse sentence meanings with propositions, and thus deny that a sentence may, without lexical or syntactic ambiguity or referential variability, express distinct propositions, an objectual necessity and a metalinguistic contingency. Or one might misconstrue logical form as a property of propositions rather than sentences, and thus deny that synonym intercepting alters logical syntax. Removing these and sundry other obstacles is a task for other essays. We're exploring the Test because it obscures recognition of the Principle's limitation to extralogical sentences. It operates with conceptions of great sophistication and charm, and offers befuddling arguments for the Principle's applicability to interceptions, arguments whose plausibility persists despite their being blatantly question begging.

We may let the Test help expose Carnapian ambitions, but when the it challenges commonsensical equivalences, only devout Churchfolk qualmlessly trust the results. While the Test passes the necessary equivalence, $B \equiv B t$ :

Bt: It is true that blood is red

it flunks their well known necessary equivalence to Bqt

Bqt: "Blood is red" is true

Why? Allegedly, Bqt translates as

Bqtg: "Blood is red" ist wahr

not as

\section{Bgqtg: "Blut ist rot" ist wahr}

Since Bqtg is logically independent of the translations of B and Bt

$\mathrm{Bg}$ : Blut ist rot

Btg: Dass Blut ist rot ist wahr 
the Test concludes Bqt isn't logically equivalent to B and Bt.

The Test reasoning is wondrously asymmetrical. It says: (1) B, Bt, Bqt are translated by Bg, $\mathrm{Btg}$, Bqtg; (2a) the truth functional equivalence of Bg and Btg to Bqtg depends on B's meaning, so (2b) Bg and Btg can be understood and known to be true without knowing the truth of Bqtg, so (2c) Bqtg is logically independent of Bg and Btg, so (3) Bqt is logically independent of B and Bt. Yet, plainly (4a) the equivalence, $B q t \equiv B \equiv B t$, is independent of B's meaning, so (4b) B and Bt cannot be understood and known to be true without knowing the truth of Bqt, just as (5a) the equivalence, $\mathrm{Bgqtg} \equiv \mathrm{Bg} \equiv \mathrm{Btg}$, is independent of Bg's meaning, so (5b) $\mathrm{Bg}$ and Btg cannot be understood and known to be true without knowing the truth of Bgqtg. So, contra (3), the equivalences in (4) and (5) must be necessary. Since (2) seems safe, the misstep must be (1). Bqt must translate as Bgqtg to reflect $B q t \equiv B \equiv B t$ 's logical form. This locates the misstep. How and why does it get taken?

The denial of the metalogical truth axiom

QT3: "..." is true $\equiv \ldots \equiv$ it is true that ...

derives from the denial of the metalogical meaning axiom, QSI: "..." means ... . The Test assumes that, to license enquotation and disquotation between Bqt and Bt, a contingent Bqs must be premised. Yet, the reasoning requiring that premise calls for another:

Bqsqs: "'Blood is red' says blood is red" says "Blood is red" says blood is red

which calls for Bqsqsqs, and so on. Denying that QSI is a metalogical principle misconstrues its inferential role in the manner Lewis Carroll explained with the object level principle, modus ponens. If $p(p \rightarrow q) \rightarrow q$ must be premised in inferences according with it, a futile regress of premises would be required.

The Test's critique of QT3 equivalences comes from its critique of QSI, but is less convincing, because QSI's semantic verb demands a linguistic expression as quotational referent, whereas a Bqt quotation is normally appositional to a term like 'proposition' denoting a translinguistic abstraction, so, normally, replicating, not translating, Bqt's enquoted sentence is unjustifiable. Replication is required only when Bqt's quotation is appositional to a language specifier as in

Bqet: The English, "Blood is red", is true

Bqet is a contingent, empirical truth, knowable only a posteriori, and rightly translated by replication as in 
Bqetg: Die englisch Satz, "Blood is red", ist wahr

Unlike Bqt, Bqet needs a premise

Bqes: The English sentence 'Blood is red' says blood is red

to form an equivalence with Bt and $\mathrm{B}: B \equiv B t \equiv(B q e s \rightarrow B q e t)$.

But generally there's no reason for assuming a modifier like that, no more than on an utterance's unenquoted words. No principle of language or rational discourse prohibits interjecting foreign words in our utterances. It's a formalist Traumerei that natural languages are formal systems wherein symbols not listed in the language's lexicon can't enter kosher sentences, except enquoted where they're displayable undefined.

Normally, absent counterindicators, when a quotation names a linguistic expression, the implicit specifier is the same as operates on every word in the utterance, and the utterance as a whole: viz., a self-referential specifier like 'in the language of this utterance': [ITLOTU]. That qualifier seems innocuous, but it's not impotent. It precludes non-self-referential specifiers that render QSI and QT3 truths empirical contingencies. It enforces reading QSI as a logical principle with QT3 as a corollary. It says Bqt's enquoted sentence replicates the unquoted sentences in $\mathrm{B}$ and $\mathrm{Bt}$, so the quotational referent is that unquoted sentence (type) — so extensional equivalence is secured syntactically, independent of which language this language is and which of its sentences is enquoted. [ITLOTU] says that, unlike Bqsg, Bqsi isn't a debatable, contentful, substantive semantic claim; it's true whatever language 'this' indexes, and whatever the enquoted is or means. Translating the self-referential [ITLOTU] of a metalogical sentence demands intraquotation translation. Replicating the enquoted is rejected, for the enquoted must replicate its utterance's unenquoted occurrences. So QSI and QT3 sentences are translatable only by QSI and QT3 counterparts, not by interceptions.

Churchfolk condemn sacrificing factual content for the sake of metalogical syntax. Although Bqs and Bgqsg are identical in syntax and predicate, they differ in objectual reference. English can express only the contingent fact that in German Bgqsq and $B g q t g \equiv B g \equiv B t g$ are logical truths; and German can state only the contingent fact that the English Bqs and $B q t \equiv B \equiv B t$ are logical truths. The only metalogical truths we can assert are those of the language we're speaking. No language neutral necessity is expressible. Metalogical reality is relative to a language. Yet this referential diremption is of minimal semantic import, because the specific referent expressions and their meanings never enter the explanation of the truth of (meta)logical sentences. As a metalogical truth, the referent is identified as and by its intrasentential replica. 
The syntactic switch of intercepting is fundamental. The semantics of intraquotation subbing is a subsidiary matter. Intercepting always alters sentence syntax and sense: whether the replica replaced is quoted or unquoted, the result is loss of logical form. The metalogical level is special because there interception can alter truth value as well as syntax and sense. Synomic intercepting of unembedded object level logical sentences converts a logical necessity into a lexical necessity. Synomic intercepting of unembedded QSI and QT3 metalogical sentences converts a logical necessity into an empirical contingency. Synomic intercepting of logical sentences embedded in extralogical sentences (Jess said "A lawyer is a lawyer"; $R W$ believes it's a logical truth that a lawyer is a lawyer) can convert a contingent truth into a falsehood. So, intercepting any logical truth embedded in a QSI sentence transforms a logical necessity into a logical impossibility:

LLqsi: "A lawyer is a lawyer" says a lawyer is a lawyer

LLqsAL: "A lawyer is a lawyer" says a lawyer is an attorney

ALqsLL: "An attorney is a lawyer" says a lawyer is a lawyer

Synomic interceptions differ semantically from logical truths - and from each other not, per se, in their objectual reference, their factual content. The difference is in what they express about their metalinguistic presuppositions, which is what gets stated in their metalinguistic equivalents, $Q S \equiv Q Q S ; Q S I \equiv Q Q S I$ :

QQS: '...' means what ' ' means

: What '...' means is what '__' means

:The meaning of '..' is the meaning of '

QQSI: What '...' means is what '...' means

More precisely, QQSI is a self-identity when the quotational qualifier recurs, whatever the qualifier be. $Q Q S I \equiv Q S I$ is a logical truth when its three quotations are qualified by [ITLOTU]. [ITLOTU] must qualify any QQS's second quotation for QQS to mutually entail its correlate QS.

Churchfolk deny the equivalence of AL (An attorney is a lawyer) to aqsl ('Attorney' means lawyer) and aqqsl (What 'Attorney' means is what 'lawyer' means) because AL is a lexical necessity while aqsl and aqqsl are contingencies. This denial doubly misconceives QS and QQS syntax. 
QQSI's logical form is unmistakably: $x=x$. So LL's ( $A$ lawyer is a lawyer) mutual entailment of lqqsi (What "lawyer" means is what "lawyer" means) is unmistakable. So too, when aqqsl's terms are read as rigidly designating the meanings actually indexed by[ITLOTU], aqqsl is an alter-identity (form $b=c$ ), a lexical necessity mutually entailing AL's lexical necessity. Those lexical necessities are explainable only by the contingency stated on the nonrigid reading of aqqsl's definite descriptions.

What obscures QS's mutual entailments (and QSI's logical truth) is QS's peculiar syntax. Paradoxically, QS's semantic verb demands a referential direct object, yet it accepts expressions from any syntactic category

tqst: 'The' means the

iqsi: 'If' means if

iqsw: 'If' (sometimes) means when

Since QS and QQS are equivalent, the QS direct object interchanges with the QQS singular term, what "_ means. That direct object is an ordinary singular term when "means" means designates (refers to), so " $D$ " means $E$ entails $D=E$. When "means" means expresses (says, predicates, connotes), $D=E$ isn't entailed and may be grammatical Unsinn. The iqsw when does not refer to the meaning of 'if' (or to anything else). Instead it there representswhat 'if' means by expressing its own meaning, expressing itself and not designating something outside itself. To model the QS syntax on QQS is to close the notional circle of nominal definitions which say only that two symbols have the same meaning without identifying that shared meaning. QQS sentences link lexical items to extralinguistic ones only via QS syntax.

The QS direct object represents its meaning by expressing it, not by designating it or being objectually about it. The syntax may seem suspect, yet the QS semantic verb simply enforces a reading that is elsewhere an available alternative to the objectual reading of utterances, an option that operates in the metalinguistic reading of synomic interceptions as QS or QQS sentences. The same autosemic (representing its meaning) option appears when utterances objectually observational, like 'This is red' ('Gavagai', etc.) are uttered as ostensive definitions expressible as: This is called "red".

The objectual reading of any utterance presupposes metalinguistic facts the utterance can express, and mutually entailing QS or QQS utterances state. B may be uttered to express the equivalents:

Blood is what is called 'red' 


\section{What is called 'blood' is red}

\section{What is called 'blood' is what is called 'red'}

Generally, the default reading of any utterance is objectual, but that reading assumes that the speaker assumes that the audience understands her metalinguistic presuppositions. A speaker says ' $p$ ' to say $p$ only if she believes that ' $p$ ' says $p$. Normally she expresses a belief that $p$ by saying ' $p$ ', whether its her belief or not. Either way, normally she expresses her belief that ' $p$ ' says $p$.

Metalinguistic readings make sense when audience understanding of the metalinguistic presuppositions is not presupposed. QS and QQS readings of synomic interceptions are natural because normally their utterance is idle if their metalinguistic presuppositions are understood.

Consequently, synonym intersubstitution between synomic interceptions must also defy the Principle. AL and AgL (An Awalt is a lawyer) aren't synonymous, because their metalinguistic presuppositions differ from each other, along with those of LL. As with synonym intercepting itself, synonym intersubbing between unembedded synonym interceptions transmits truth. Obviously, subbing into any enquoted sentence needn't preserve the enquoting sentence's truth value when translation requires quotational replication, as in: Ed said "An attorney is a lawyer". Less obviously, subbing into belief attributions (and their consequent attitudinal attributions) needn't preserve the embedding utterance's truth value because the logical and interceptional utterances' metalinguistic presuppositions are epistemically independent.

Finally, note the reflection of QSI's autosemic syntax in its corollary, TF5. B also mutually entails

Bf: It is a fact that blood is red

and thus also

Bnf: Blood's being red is a fact

Thus QT3 is only 3/5's of:

TF5: 'NP VP' is true $\equiv$ It's true that NP VP $\equiv \mathrm{NP}$ VP $\equiv$

It's a fact that NP VP $\equiv$ NP's VPing is a fact

Yet Bqf and Bnt are nonsense. 
Bqf: 'Blood is red' is a fact

Bnt: Blood's being red is true.

So, in Bf, B functions like a nominalization of the sentence, but represents the fact autosemically: it expresses the fact, but without referring to it as the nominalization does. In Bt, B functions like a name of the proposition, but represents the proposition autosemically: it expresses the proposition without referring to it as Bqt does.

\section{Notes}

(1) See Alonzo Church, 1950, "Carnap's Analysis of Statements of Assertion and Beliefs," Analysis 10, 5: 97-99; and 1954, "Intensional Isomorphism and Identity of Belief", Philosophical Studies 5; 65-73.

(2) See my "Identity Syntax", Proceedings of the 20th World Congress of Philosophy, and "Identity: Logic, Ontology, Epistemology", Philosophy, (78), 1998.

(3) We may, elliptically and misleadingly, call quotations names. Yet, strictly, neither the markers, nor the enquoted material, nor their combination is a referring expression. The markers signal that, whatever other functions the enquoted material may be serving, that material is being displayed, like a sentence embedded color patch, to facilitate reference to something identifiable with it. A quotation operates in apposition to an explicit or implicit referring expression, usually a description. If there weren't an implicit apposition to something like "The letter sequence", then 1 and 2

1: "Qkxf" is not a meaningful expression

2: "The" is not a referring expression would be ungrammatical or necessarily false, and 3 and 4 wouldn't be sentences at all:

3. 'The' is 'the'

4. "Mother" is "Mother"

Parmenedian puzzles about denials (and assertions) of existence have metalogical parallels. See my "Quotation Apposition", forthcoming in Analysis.

(4) If synonyms are semantically equivalent expressions, then, in its use as a replicator, the synonyms of a quotation are replications of the enquoted material marked by alternative devices, like italics or underlining. Subbings of such 'synonyms' conform to the Principle. 
(5) See my The Significance of Sense (Cornell University Press, Ithaca) 1972, esp. Ch. 2, and "Synonymy Without Analyticity", International Philosophical Preprint Exchange, Nov., 1994

(6) See my "Distinguishing Mathematics From Logic", Proceedings of the 20th World Congress of Philosophy, and "How Mathematics Isn't Logic", forthcoming in Ratio, 1999.

(7) See, in addition to works cited above, my "The Synonymity Antinomy" and "Quotation Translation", forthcoming.

(8) Now compare: QSI translations are isomorphic in all but objectual reference. Synomic interceptions of (object level) logical sentences are simply untranslatable (except by importing the terms) since their factual content is only an artifact of internotational contingencies. The extralogical QS and QQS sentences are fully translatable by quotation replication. So too for QQSI sentences, for they are metalogical only in their terms, not in their syntax. However, the equivalence of a QS/QSI sentence to its QQS/QQSI correlate is a metalogical truth, untranslatable by quotation replication.

(9) Or perhaps Bnf is: The redness of blood is a fact. The proper nominal form for facts is not here at issue. 